\title{
An Unusually Giant Hematoma threatening to the Laryngeal Airway
}

\author{
${ }^{1}$ Santosh Kumar Swain, ${ }^{2}$ Sidhartha Mohanty, ${ }^{3}$ Neha Singh, ${ }^{4}$ Rankanidhi Samal
}

\begin{abstract}
An acute onset of giant laryngeal hematoma is rare and lifethreatening condition. It occurs usually in patients with coagulopathies, often in those receiving warfarin. Various sites of bleeding have been reported in the literature, most commonly being the gastrointestinal tract, genitourinary tract and central nervous system. A large acute onset spontaneous hematoma is rarely reported in larynx. We report a case of an unusually large hematoma compromising the glottic airway in an over warfarinised patient. We highlight the need for a thorough evaluation of upper aerodigestive tract in patients on anticoagulant therapy. Detailed airway evaluation and monitoring with flexible laryngoscopy, and timely needful intervention are essential in above type of patients for preventing life-threatening consequences.
\end{abstract}

Keywords: Airway, Hematoma, Larynx, Warfarin.

How to cite this article: Swain SK, Mohanty S, Singh N, Samal R. An Unusually Giant Hematoma threatening to the Laryngeal Airway. Int J Otorhinolaryngol Clin 2014;6(3):92-94.

Source of support: Nil

Conflict of interest: None

\section{INTRODUCTION}

Hematoma in the airway is rare and life-threatening situation. The origin of hematoma may be spontaneous or may be precipitated by trauma. Over anticoagulation enhances the risk of hemorrhage and formation of spontaneous hematomas. Drug interactions are common cause for over anticoagulation. Various sites of bleeding with hematoma formation have been reported in the literature, most commonly being the gastrointestinal tract, genitourinary system and central nervous system. The upper airway may also be affected, sometimes with life-threatening situations. ${ }^{1}$ Spontaneous hematoma formation at the upper aerodigestive tract is rarely reported and being potentially a life-threatening condition. Here, we are

${ }^{1}$ Associate Professor, ${ }^{2,4}$ Professor, ${ }^{3}$ Assistant Professor

${ }^{1,4}$ Department of ENT, Institute of Medical Sciences and SUM Hospital, Bhubaneswar, Odisha, India

${ }^{2,3}$ Department of Anesthesia, Institute of Medical Sciences and SUM Hospital, Bhubaneswar, Odisha, India

Corresponding Author: Santosh Kumar Swain, Associate Professor, Department of ENT, Institute of Medical Sciences and SUM Hospital, Bhubaneswar, Odisha, India, Phone: 06742386292 xe-mail: swainsantoshbbsr@yahoo.com presenting a case with spontaneously formed large hematoma arising from posterior wall of interarytenoid area after taking warfarin which was threatening to the glottic airway and at the same time causing dysphagia.

\section{CASE REPORT}

A 56-year-old lady, presented to ENT OPD, IMS and SUM Hospital, Bhubaneswar, Odisha, India with complaints of plumy voice and dysphagia to solid foods since 5 days. She has mild dyspnea. The clinical manifestations are sudden in onset and progressive in nature. There was no history of trauma to the neck and she was not suffering from any bleeding disorder. On clinical examination, i.e. indirect laryngoscopy examination, a smooth bluish to black color mass arising from the posterior part of interarytenoid area and blocking the glottic airway. She was hemodynamically stable. Her blood pressure was 140/ $70 \mathrm{~mm} \mathrm{Hg}$ with antihypertensive medications. The patient had been receiving warfarin for atrial fibrillation since 1 month prescribed by local physician. Onset of plumy voice and dysphagia were sudden in nature and manifested 1 month after starting warfarin. On examinations ear, nose, oral cavity and oropharynx were within normal limit. Flexible laryngoscopy revealed an unusually large hematoma present submucosally completely blocking the glottic airway (Fig. 1). The mass was pedunculated attached to the posterior wall of interarytenoid area. At its normal position, it blocked the postcricoid area, causing dysphagia to the patient and the laryngeal airway was free. During flexible laryngoscopy airway stimulation caused the pedunculated mass to come anteriorly and block the glottic airway temporarily. Pulse oximetry showed fluctuating levels of $\mathrm{O}_{2}$ saturation between 85 to $90 \%$. After hospitalization, patient had no sign of breathing difficulty. She received nebulization with steroids and bronchodilators. After nebulization, the appearance of the mass changed to pale and dusky on repeat flexible laryngoscopy (Fig. 2). Coagulation tests were grossly abnormal (PT: 90 secs, INR: 10.4, APTT: 70 secs) at the time of hospitalization. Warfarin was stopped and vitamin K was administered intravenously. After 72 hours improvement of coagulation profile occurred. Preoperative investigations were done after admitting the patient in hospital in view to secure the airway by operative intervention for such a large mass. CT scan of the neck was done for knowing the origin of mass and extent of involvement (Fig. 3). 


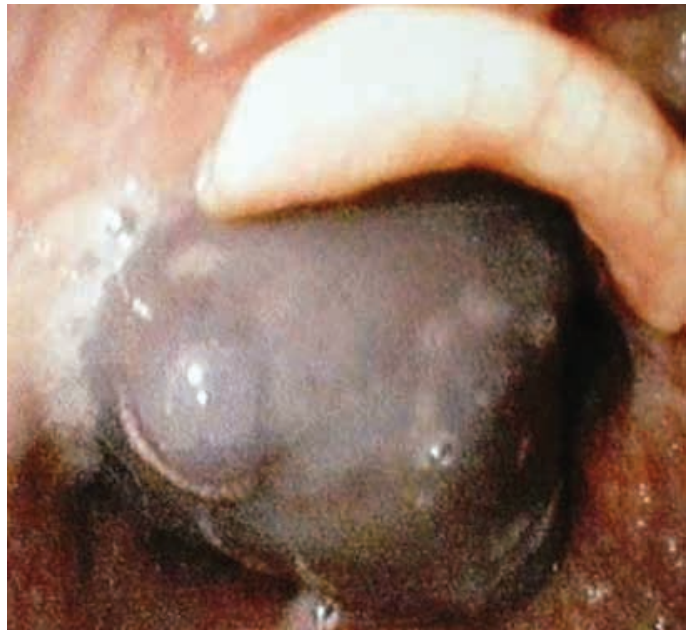

Fig. 1: A giant hematoma at laryngeal airway

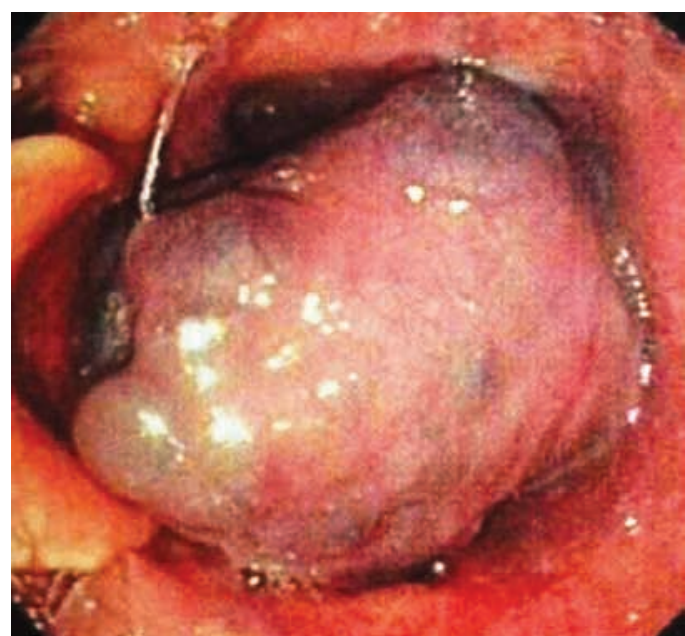

Fig. 2: A dusky colored hematoma at laryngeal airway after repeated nebulization

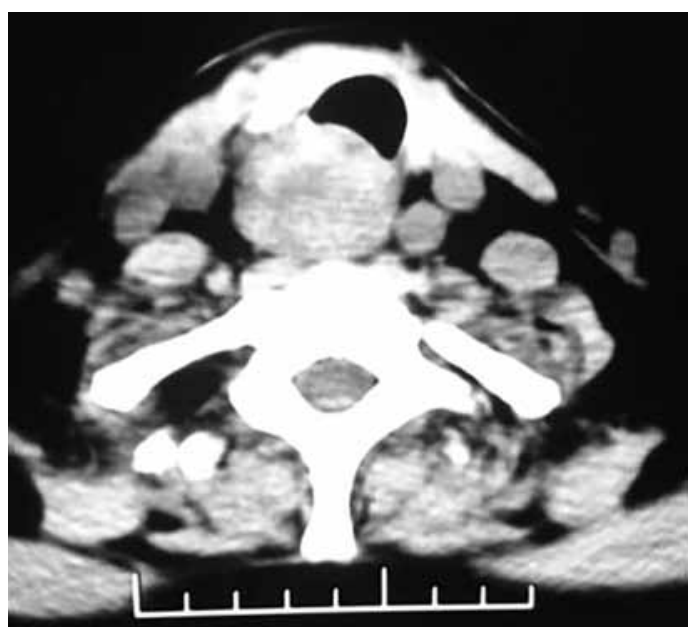

Fig. 3: Computed tomography scan showing laryngeal hematoma

The CT report showed increased vascularity which was suggesting vascular mass or hematoma. Under general anesthesia, awake nasotracheal intubation was done by fiberoptic bronchoscopy, after nebulization with $4 \%$ lidocaine. A smaller size $(5.5 \mathrm{~cm}$ size) endotracheal tube was introduced with great care under vision without producing any injury to the mass. Microlaryngoscopic excision of the mass was done. The mass was meticulously removed from its base with the help of microscissors without injuring vocal cords. Bleeding was very minimal. The hemostasis was achieved. Altered blood and clots were seen on dissection of the mass after removal. The mass was sent for histopathological examination.

Histopathological examination showed old clotted blood present in the sac lined by mucosa reported as hematoma. The recovery was uneventful. Patient was extubated once fully awake. There was no evidence of any residual mass or recurrence after 6 months of surgery.

\section{DISCUSSION}

An acute spontaneous hematoma occurring at upper airway is an uncommon but potentially a life-threatening event. Sometimes it occurs among patients being treated with anticoagulants as well as in those patients with coagulation defects, such as hemophilia. Our patient developed laryngeal hematoma after taking warfarin for 1 month. She had taken warfarin after diagnosis of atrial fibrillation by local physician. Warfarin is a commonly used anticoagulant which acts by antagonising the action of vitamin $\mathrm{K}$; which is a cofactor for synthesis of coagulation factors II, VII, IX, X and protein C and $S^{2}$ The anticoagulative effect of warfarin can be compounded by dysfunction in other procoagulant mechanisms, such as decreased absorption of vitamin $\mathrm{K}$ and platelet abnormalities. In daily otolaryngology practice, this commonly presents as nasal bleeding. Other specialties manage different manifestations, such as subconjunctival hemorrhage, hematuria and melaena. Intracranial and gastrointestinal hemorrhage can be fatal. Hematoma occurring in the upper airway is rare and prove serious. The main risk of oral anticoagulants is always hemorrhage. Minor gingival bleeding, traumatic purpura, or trace hematuria may occur even within the therapeutic range of international normalized ratio (INR). ${ }^{3}$ Elderly patients or patients with past history of stroke, gastrointestinal hemorrhage, first atrial fibrillation episode, concomitant use of aspirin or other comorbidity like diabetes, anemia, uremia or hepatic disease and with infrequent monitoring of the INR, carry higher risk. ${ }^{4}$ Spontaneous hemorrhage may occur in different sites of the body, more commonly in gastrointestinal tract, genitourinary tract, central nervous system, nose, retroperitoneum, subcutaneous tissues and wounds. Involvement of upper aerodigestive region causing obstruction of the laryngeal airway which is a rare and life-threatening complication. ${ }^{5}$ Our case had glottic airway obstruction with giant hematoma arising from posterior wall of interarytenoid area and it is going 
posteriorly and blocking postcricoid region so causing dysphagia.

Airway hematoma is a rare and life-threatening complication of warfarin therapy. Warfarin is commonly used anticoagulants with indications such as atrial fibrillation and artificial heart valves. It has been seen that there is a $6.8 \%$ incidence of hemorrhage in patients taking warfarin. ${ }^{6}$ Early identification of this condition can allow successful conservative treatment and avoid airway intervention. There are different etiology for airway hematomas have been postulated, e.g. result of local trauma (vigorous sneezing or coughing) or infection., Airway hematomas may be seen in sublingual and retropharyngeal spaces, ${ }^{9}$ but it is less commonly seen in the larynx. Most of the cases are managed conservatively with oxygen, reversal of the coagulopathic state, parenteral steroids and airway observation.

A suspected hematoma of the laryngeal airway should be confirmed by indirect laryngoscopy or flexible laryngoscopy performed by an experienced otolaryngologist. The use of computed tomography (CT) scan in the laryngeal hematoma is effective when a flexible laryngoscopy is limited by airway edema. Initial conservative management of laryngeal hematoma with mild symptoms are close observation and reversal of anticoagulation with parenteral administration of vitamin $\mathrm{K} .{ }^{10}$ In our case, the mass was very large threatening the laryngeal airway, needed urgent surgical intervention. Patient had undergone immediate microlaryngeal drainage of hematoma with careful nasotracheal intubation by a team of experienced anesthetists.

\section{CONCLUSION}

This case highlights the potential dangers of warfarin therapy, and how rapidly an laryngeal hematoma can become life-threatening. We suggest recommendation of this diagnosis in patients taking anticoagulants or in coagulopathic patients. They need thorough endoscopic airway evaluation, frequent observations for clinical features pertaining to airway obstruction and timely management of the above complications early.

\section{REFERENCES}

1. Kerr HD, Kwaselow A. Vocal cord hematomas complicating anticoagulant therapy. Ann Emerg Med 1984;113(7):552-553.

2. Hughes J, Mulki O, Stephens J, Robinson J. Extensive laryngopharyngeal haematoma in warfarinised patient requiring emergency tracheotomy. Internet J Otorhinolaryngol 2007:8(1).

3. Mosher DF. Disorders of blood coagulation. Part XIII: Hematological diseases. In: Bennett JC, Plum F, editors. Cecil Textbook of Medicine. 20th ed. WB Saunders Co, Philadelphia; 1996;999-1000.

4. Harker LA. Antithrombotic Therapy. Part V: Principles of evalution and management. In: Bennett JC, Plum F, editors. Cecil Textbook of Medicine. 20th ed. WB Saunders Co: Philadelphia; 1996;120-121.

5. Gonzalez-Garcva R, Schoendorff G, Mupoz-Guerra MF, Rodrvguez-Campo FJ, Naval-Gvas L, Sastre-Purez J. Upper airway obstruction by sublingual haematoma: a complication of anticoagulation therapy with acenocoumarol. Am J Otolaryngol 2006;27(2):129-132.

6. Coon WW, Willis PW 3rd. Hemorrhagic complications of anticoagulant therapy. Arch Intern Med 1974 Mar;133(3): 386-392.

7. Jandreau SW, Mayer D. Spontaneous bilateral arytenoids hematoma in a patient on warfarin. Am J Emerg Med 1998 Nov;16(7):674-676.

8. Lim M, Chaudhari M, Devesa PM, Waddell A, Gupta D. Management of upper airway obstruction secondary to warfarin therapy: the conservative approach. J Laryngol Otol 2006 Feb;120(2):e12.

9. Ako E, Seyfeli E, Ako S, Karazineir S, Okuyucu S, Da AS. Retropharyngeal hematoma as a complication of anticoagulation therapy. Ear Nose Throat J 2008 Mar;87(3): $156-159$.

10. Triaridis S, Tsiropoulos G, Rachovitsa D, Psillas G, VitalV. Spontaneous haematoma of the pharynx due to a rare drug interaction. Hippokratia 2009;13(3):175-177. 